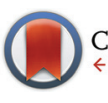

CrossMark

\& click for updates

Cite this: Polym. Chem., 2015, 6 , 8036

Received 31st July 2015,

Accepted 23rd September 2015

DOI: $10.1039 / c 5 p y 01210 d$

www.rsc.org/polymers

\section{Interactions of a zwitterionic thiophene-based conjugated polymer with surfactants $\uparrow$}

\author{
Telma Costa, ${ }^{\text {a }}$ Diego de Azevedo, ${ }^{a}$ Beverly Stewart, ${ }^{a}$ Matti Knaapila, \\ Artur J. M. Valente, ${ }^{a}$ Mario Kraft, ${ }^{c}$ Ullrich Scherf ${ }^{c}$ and Hugh D. Burrows ${ }^{a}$
}

In this paper we investigate the optical and structural properties of a zwitterionic poly[3-( $N$ - (4sulfonato-1-butyl)-N,N-diethylammonium)hexyl-2,5-thiophene] (P3SBDEAHT) conjugated polyelectrolyte (CPE) and its interaction in water with surfactants, using absorption, photoluminescence (PL), electrical conductivity, molecular dynamics simulations (MDS) and small-angle X-ray scattering (SAXS). Different surfactants were studied to evaluate the effect of the head group and chain length on the self-assembly. PL data emphasize the importance of polymer-surfactant electrostatic interactions in the formation of complexes. Nevertheless, conductivity and MDS data have shown that nonspecific interactions also play an important role. These seem to be responsible for the spatial position of the surfactant tail in the complex and, eventually, for breaking-up P3SBDEAHT aggregates. SAXS measurements on P3SBDEAHT-zwitterionic cocamidopropyl betaine (CAPB) surfactant complexes showed a specific structural organization of the system. The CAPB surfactant promotes a structural transition from pure P3SBDEAHT 3-dimensional aggregates (radius of gyration $\sim 85 \AA$ ) to thick cylindrical aggregates ( 20 Å) where all CAPB molecules are associated with the polymer. For molar ratios (in terms of the polymer repeat unit) $>1$ the SAXS interference maximum of the complexes resembles that of pure CAPB thus suggesting ongoing phase segregation in the formation of a "pure" CAPB phase.

\section{Introduction}

Water-soluble conjugated polythiophene (PT)-based polyelectrolytes are an important class of conjugated polymers which have received great attention as active materials in optoelectronic devices and in biosensing. ${ }^{1}$ PT derivatives show unique chromic features that can be used as simple, rapid, versatile and sensible detection tools. The colour of PT solutions can vary from deep violet $\left(\lambda_{\max } \sim 550 \mathrm{~nm}\right)$ to bright yellow $\left(\lambda_{\max } \sim 400 \mathrm{~nm}\right){ }^{2}$ The changes in $\pi-\pi^{*}$ transitions are related to conformational changes of the backbone from planar to nonplanar. Conformational changes can be promoted by changing temperature, $\mathrm{pH}$, ionic strength, cations, anions, surfactants, nucleic acids, proteins, etc. ${ }^{2-10} \mathrm{~A}$ detailed study of the optical transitions of a water-soluble PT induced

${ }^{a}$ Centro de Química de Coimbra, Departamento de Química, Faculdade de Ciências e Tecnologia, Universidade de Coimbra, 3004-535 Coimbra, Portugal.

E-mail: tcosta@qui.uc.pt

${ }^{b}$ Department of Physics, Technical University of Denmark, $2800 \mathrm{Kgs}$. Lyngby, Denmark ${ }^{c}$ Macromolecular Chemistry Group, Bergische Universität Wuppertal, D-42119 Wuppertal, Germany

$\dagger$ Electronic supplementary information (ESI) available. See DOI: 10.1039/ c5py01210d by surfactant addition was performed by Knaapila and coworkers. ${ }^{8,9}$ The optical transitions are correlated with the changes in the type of CPE-surfactant aggregate formed in solution. The phase behavior of two cationic PTs with different pendent groups, poly[3-(6-( $N$-methylimidazolium)hexyl)-2,5thiophene] (P3ImiHT) ${ }^{9}$ and poly[3-(6-trimethylammoniumhexyl)thiophene]bromide (P3TMAHT) ${ }^{8}$ was investigated in the presence of sodium dodecylsulfate (SDS). In pure $\mathrm{D}_{2} \mathrm{O}$ solutions, P3TMAHT forms charged aggregates with interparticle order. The presence of SDS leads to phase transitions from rod-like $(x=1 / 5)$ to sheet-like particles $(x=1 / 2$ to 1$)$ with embedded polymer bundles ( $x=1 / 5$ to $1 / 2)$ or sheet-like $(x=1)$ polymer associations, to ellipsoidal particles for $x \approx 5$. These phase transitions occur with concomitant color transitions: pale red (Р3ТМAHT) to turbid violet ( $x=1 / 5$ to 1 ) and orangeyellow $(x>2)$. Similar, but not identical, behavior is observed for P3ImiHT. The main difference between the P3TMAHT $(\mathrm{SDS})_{x}$ and P3ImiHT(SDS) $)_{x}$ systems is the occurrence of precipitation at the charge compensation point in the former system. The more bulky $N$-methylimidazole end groups of P3ImiHT, in relation to the smaller trimethylammonium groups of P3TMAHT, do not allow full charge compensation, and, hence, prevent precipitation. 
Zwitterionic CPEs (ZCPEs) show advantages over regular, cationic or anionic CPEs due to the absence of mobile counterions. It has been shown that the size of the counterion can have a strong effect on the optical properties of CPEs. The UV/vis absorption spectra of 2,5-poly(thiophene-3-propionic acid) shifts over a $130 \mathrm{~nm}$ range by changing the counterion. ${ }^{11}$ This may be an advantage in some cases, since one can modulate the degree of aggregation in solution and hence its optical properties. Nevertheless, mobile counterions may also have negative effects on the performance of electronic devices as OLEDs or OFETs, due to luminescence quenching or alterations of work function of electrodes. Interlayers of zwitterionic CPEs can improve the electron injection and thus improve device performance. ${ }^{12-15}$ An OLED device containing an electrode interlayer of zwitterionic poly[(9,9-bis( $N$-(4-sulfonato1-butyl)- $N, N$-dimethylammonium)ethyl)-2,7-fluorene)-alt-2,7(9,9-dioctylfluorene)] showed a very short response time (less than $10 \mu \mathrm{s})$. The localized nature of all ions near the cathodes facilitates charge injection, the current efficiency was enhanced by a factor of two. ${ }^{12}$ Zwitterionic polythiophene and poly(thiophene-alt-benzothiadiazole) derivatives were used as interfacial modification layers in organic photovoltaic devices and they greatly improved the power conversion efficiency (PCE) of organic solar cell (OSC) devices with $\mathrm{Al}$ or $\mathrm{Ag}$ cathodes. ${ }^{16}$ The PCE was enhanced $>50 \%$ for an $\mathrm{Ag}$ cathode and $68 \%$ for an $\mathrm{Al}$ cathode, respectively.

ZCPEs also show promise for applications as sensing platforms. A polar polythiophene carrying amino acid side chains (POWT) can interact with positively or negatively charged peptides, as well as with four-helix bundles that were formed by the two peptide chains. ${ }^{17}$ Different polythiophene-peptide complexes are formed, with characteristic optical features. The differences are due to changes in the effective conjugation length and/or due to interchain interactions and aggregation effects. Similar effects are also found when POWT interacts with ss-DNA or ds-DNA. ${ }^{18,19}$ Hence, a detailed characterization of the ZCPE structure in the solid or (nano)aggregated state seems crucial in the development of new sensing platforms. In this study, we report the behavior of the zwitterionic P3SBDEAHT alone on water, and with various types of surfactants.

\section{Experimental}

\section{Materials}

The synthesis of the water soluble poly[3-( $N$-(4-sulfonato1-butyl)- $N, N$-diethylammonium)hexyl-2,5-thiophene) (P3SBDEAHT) was carried out in analogy with the synthesis of similar polyfluorene-based ZCPEs as described in ref. 12 and 13. The detailed experimental procedure for P3SBDEAHT synthesis is given in the ESI. $\dagger$ The synthesis includes a two-step postpolymerization functionalization (Scheme 1) of the well-known precursor polymer poly(3-bromohexyl)thiophene (P3BrHT). ${ }^{20,21}$ Hereby, P3BrHT with a narrow molecular weight distribution

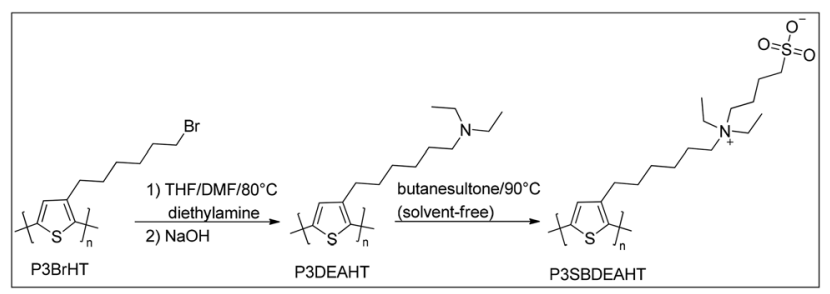

Scheme 1 Synthesis of the conjugated, zwitterionic polyelectrolyte poly[3-( $N$-(4-sulfonato-1-butyl)- $N, N$-diethylammonium)hexyl-2,5-thiophene) (P3SBDEAHT).

was made in a so-called Grignard metathesis polymerization procedure (GRIM). ${ }^{22}$

The P3BrHT sample used in the synthesis of P3SBDEAHT showed a number average molecular weight $\left(M_{\mathrm{n}}\right)$ of $11500 \mathrm{~g}$ $\mathrm{mol}^{-1}$ (corresponding to a degree of polymerization (DP) of 47) and a polydispersivity index (PDI) of 1.1. The detailed experimental procedure for P3SBDEAHT synthesis is outlined in the ESI. $\dagger$

All the surfactants were purchased and used without any further purification, except for cocamidopropyl betaine (CAPB). CAPB was extracted from Dehyton ${ }^{\circledR}$ K 35 (BASF) through sodium chloride precipitation with ethanol. Sodium octyl sulfate (SOS), sodium dodecyl sulfate (SDS), sodium tetradecyl sulfate (STS), pentaethylene glycol dodecyl ether $\left(\mathrm{C}_{12} \mathrm{E}_{5}\right)$ and hexadecyl trimethylammonium bromide (CTAB) were purchased from Sigma; dodecyl trimethylammonium chloride (DTAC) was purchased from Fluka. Milli-Q water was used in all solutions and the experiments were performed at the natural $\mathrm{pH}$ of the prepared solution.

\section{Absorption spectra}

The UV/visible absorption spectra were recorded on a Shimadzu UV-2100 spectrophotometer; all spectra were acquired with a minimum resolution of $0.2 \mathrm{~nm}$.

\section{Steady-state fluorescence}

The fluorescence spectra were recorded on a Horiba-JobinYvon SPEX Fluorog 3-22 spectrometer and were corrected for the instrumental response. The Fluorolog consists of a modular spectrofluorimeter with double grating excitation (range 200-950 nm, optimized in the UV and with an angle blazed at $330 \mathrm{~nm}$ ) and emission (range 200-950 nm, optimized in the visible and with an angle blazed at $500 \mathrm{~nm}$ ) monochromators. The bandpass for excitation and emission is $0-15 \mathrm{~nm}$ (values that are continuously adjustable from computer Datamax/32 software) and the wavelength accuracy is $\pm 0.5 \mathrm{~nm}$. The excitation source consists of an ozone-free $450 \mathrm{~W}$ xenon lamp and the emission detector is a Hamamatsu R928 Photomultiplier (200-900 nm range), cooled with a Products for Research thermoelectric refrigerated chamber (model PC177CE005), or a Hamamatsu R5509-42 (900-1400 nm range), cooled to $193 \mathrm{~K}$ in a liquid nitrogen chamber (Products 
Table 1 Critical micelle concentration in the absence ${ }^{40}(\mathrm{cmc})$ and presence of P3SBDEAHT (cac* and cac), maximum absorption $\left(\lambda_{\text {abs }}\right)$ and emission $\left(\lambda_{\mathrm{em}}\right)$ wavelengths, and fluorescence quantum yields $\left(\phi_{\mathrm{F}}\right)$ of P3SBDEAHT in water and in aqueous micellar solutions

\begin{tabular}{|c|c|c|c|c|c|c|c|}
\hline Solvent & $\operatorname{cac}^{*}(\mathrm{M})$ & cac (M) & cmc (M) & $C_{\text {surf }}^{a}(\mathrm{M})$ & $\lambda_{\mathrm{abs}}(\mathrm{nm})$ & $\lambda_{\mathrm{em}}(\mathrm{nm})$ & $\phi_{\mathrm{F}}{ }^{b}$ \\
\hline Water & - & - & - & - & 452 & 600 & $0.04 \pm 0.002$ \\
\hline Water & - & - & - & - & 452 & 600 & $0.043 \pm 0.002$ \\
\hline SDS & $1.16 \times 10^{-3}$ & $5.91 \times 10^{-3}$ & $8.3 \times 10^{-3}$ & $1.0 \times 10^{-2}$ & 433 & 574 & $0.113 \pm 0.012$ \\
\hline STS & $2.94 \times 10^{-4}$ & $2.07 \times 10^{-3}$ & $2.1 \times 10^{-3}$ & $3.4 \times 10^{-3}$ & 423 & 575 & $0.066 \pm 0.003$ \\
\hline CAPB & & $8.1 \times 10^{-5}$ & $9 \times 10^{-5}$ & $1.1 \times 10^{-4}$ & 444 & 593 & $0.054 \pm 0.006$ \\
\hline DTAC & & $1.96 \times 10^{-2}$ & $2.03 \times 10^{-2}$ & $2.4 \times 10^{-2}$ & 443 & 594 & $0.053 \pm 0.05$ \\
\hline
\end{tabular}

${ }^{a}$ Surfactant concentration at which the $\lambda_{\mathrm{abs}}, \lambda_{\mathrm{em}}$ and $\phi_{\mathrm{F}}$ were obtained. ${ }^{b} \phi_{\mathrm{F}}$ values were measured relative to $\alpha 5$ in methylcyclohexane $\left(\phi_{\mathrm{F}}=0.33\right)^{41}$

for Research model PC176TSCE-005), and a photodiode as the reference detector.

\section{Electrical conductivity}

Electrical resistances of the solutions were measured with a Wayne-Kerr model 4265 automatic LCR meter at $1 \mathrm{kHz}$. A diptype conductance cell with a cell constant of $11.78 \mathrm{~m}^{-1}$, and an uncertainty of $0.02 \%$, was used. ${ }^{23}$ The cell constant was determined from measurements with $\mathrm{KCl}$ (reagent grade, recrystallized, and dried) using the procedure and data of Barthel et $a .^{24}$ Measurements were taken at temperatures ranging from 298.15 to $313.15 \mathrm{~K}( \pm 0.02 \mathrm{~K})$ in a Thermo Scientific Phoenix II B5 thermostat bath.

\section{SAXS}

SAXS measurements were carried out at the I911 beamline at the MAX IV Laboratory, Lund (Sweden). The X-ray wavelength was $0.91 \AA$, and the sample-to-detector distance was $1919.8 \mathrm{~mm}$, yielding a usable $q$ range of $0.008-0.3 \AA^{-1}$. The beam size was $0.3 \mathrm{~mm} \times 0.3 \mathrm{~mm}$ (vertical $\times$ horizontal). The scattering patterns were measured using a PILATUS $1 \mathrm{M}$ detector. The scattering intensity was considered to scale as $\sim q^{-\alpha}$, where the exponent $\alpha$ was interpreted in terms of particle shape such that $\alpha=1$ implies separated cylindrical particles and $\alpha=4$ implies smooth 3-dimensional particles. When the data leveled off at low $q$, this simple interpretation was enhanced by fitting to the arbitrary or cylindrical particle shapes using an indirect Fourier transformation program GNOM. $^{25}$

\section{Molecular dynamics simulations}

Molecular dynamics simulations were performed using the GROMACS software package with the standard GROMOS96 $53 \mathrm{a} 6$ force field. ${ }^{26-28}$ Structures for P3SBDEAHT and all surfactants were built and minimized using the AVOGADRO molecular builder, ${ }^{29}$ the protein databank files of minimised structures were then submitted to the Automated Topology Builder $^{30}$ to generate the necessary topology files for simulations using GROMACS. Using molecular dynamics simu- lations a number of simulation cells were created in order to examine the aggregation of P3SBDEAHT in surfactant environments, and details of cell contents are shown in Tables S1S3. $\dagger$ Initially simulations were carried out using a $1: 1$ ratio of P3SBDEAHT: surfactant, 1a-1g. The optimised structure of P3SBDEAHT used in all simulations was the tetrameric form this was due to an atom number limit present when generating topology files using the Automated Topology Builder. ${ }^{30}$ This was followed by simulations using 1:2 P3SBDEAHT: surfactant, 2a-2g and $1: 3$ P3SBDEAHT : surfactant, 3a-3g.

The required species were added to a cubic box of $7 \mathrm{~nm} \times$ $7 \mathrm{~nm} \times 7 \mathrm{~nm}$ in accordance with the specifications given in Tables 1-3. The remainder of the cell was taken up by water by employing the SPC solvation model $^{31}$ which considers a simple 3 point charge model for water where the intramolecular degrees of freedom are frozen and the intermolecular interactions are described by a combination of Lennard-Jones and Coulombic potentials between sites of fixed point-charges. The box was then constrained in accordance with the LINCS algorithm. ${ }^{32}$ Simulations were carried out over a time frame of $20 \mathrm{~ns}$ with a step size of 2 fs. All visualisations and images were generated using VMD software. ${ }^{33}$ The SPC model used represents, with accuracy, the properties of a bulk water environment under standard conditions, $300 \mathrm{~K}$ and 1 atmosphere pressure. It has also been employed in the study of similar CPEs in aqueous environments. ${ }^{34}$

\section{Results and discussion}

\section{Absorption and fluorescence data}

The zwitterionic CPE P3SBDEAHT in aqueous solutions is characterized by a non-resolved absorption band peaking at $445 \mathrm{~nm}$ (data not shown), and a broad and featureless emission band with a maximum at ca. $600 \mathrm{~nm}$ (Fig. 1). In mixtures of water and a water-miscible organic solvent, such as dioxane, both emission intensity and the position of the emission maximum change with respect to the aqueous solution. The presence of the co-solvent dioxane leads to an increase in PL intensity and a blue shift of the PL band. When the dioxane 
Table 2 Critical micelle concentration in the absence $(\mathrm{cmc})$ and presence (cac) of P3SBDEAHT polymers, degree of dissociation ( $\alpha$ ) and standard Gibbs free energy of micellization $\left(\Delta \mathrm{G}_{\text {mic }}{ }^{\circ}\right.$ ) of SDS, CTAB and P3SBDEAHT/surfactant systems in aqueous solutions, at $25^{\circ} \mathrm{C}$

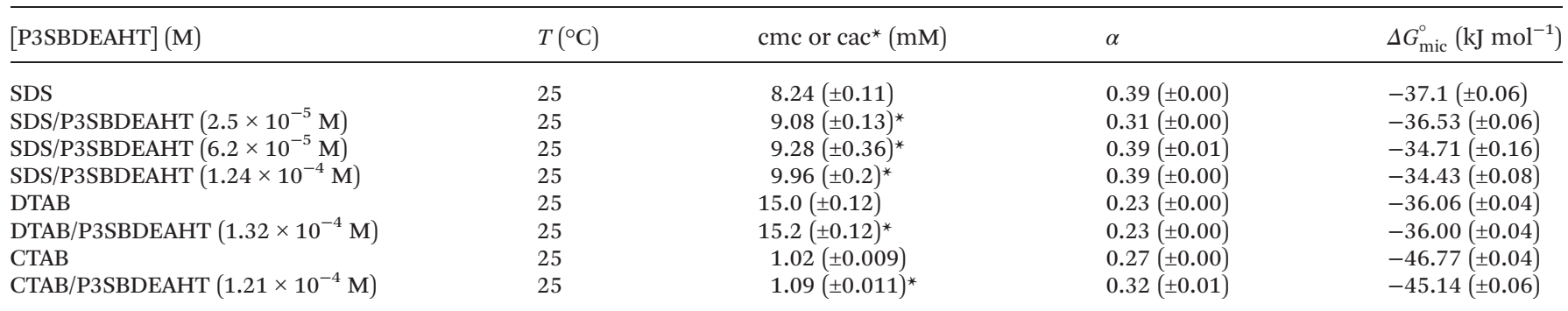

Table 3 Critical micelle concentration in the absence $(\mathrm{cmc})$ and presence (cac*) of P3SBDEAHT polymers $\left(6.3 \times 10^{-5} \mathrm{M}\right)$, degree of dissociation $(\alpha)$, standard Gibbs free energy of micellization $\left(\Delta G_{\text {mic }}{ }^{\circ}\right)$ and Gibbs free energy of micellization $\left(\Delta G_{\text {mic }}\right)$ of SDS, P3SBDEAHT/SDS systems at three different temperatures

\begin{tabular}{|c|c|c|c|c|c|}
\hline [P3SBDEAHT] (M) & $T\left({ }^{\circ} \mathrm{C}\right)$ & $\mathrm{cmc}$ or cac* $(\mathrm{mM})$ & $\alpha$ & $\Delta G_{\text {mic }}^{\circ}\left(\mathrm{kJ} \mathrm{mol}^{-1}\right)$ & $\Delta G_{\text {mic }}\left(\mathrm{J} \mathrm{mol}^{-1}\right)$ \\
\hline SDS & 25 & $8.24( \pm 0.1)$ & $0.39( \pm 0.00)$ & $-35.27( \pm 0.05)$ & \\
\hline SDS/P3SBDEAHT & 25 & $9.28( \pm 0.36)^{*}$ & $0.39( \pm 0.01)$ & $-34.7( \pm 0.16)$ & 294 \\
\hline SDS/P3SBDEAHT & 30 & $8.14( \pm 0.25)^{*}$ & $0.30( \pm 0.01)$ & $-37.19( \pm 0.13)$ & -15.8 \\
\hline SDS & 40 & $8.22( \pm 0.1)$ & $0.39( \pm 0.04)$ & $-35.15( \pm 0.05)$ & \\
\hline SDS/P3SBDEAHT & 40 & $7.87( \pm 0.1)^{*}$ & $0.41( \pm 0.00)$ & $-34.96( \pm 0.05)$ & -114 \\
\hline
\end{tabular}
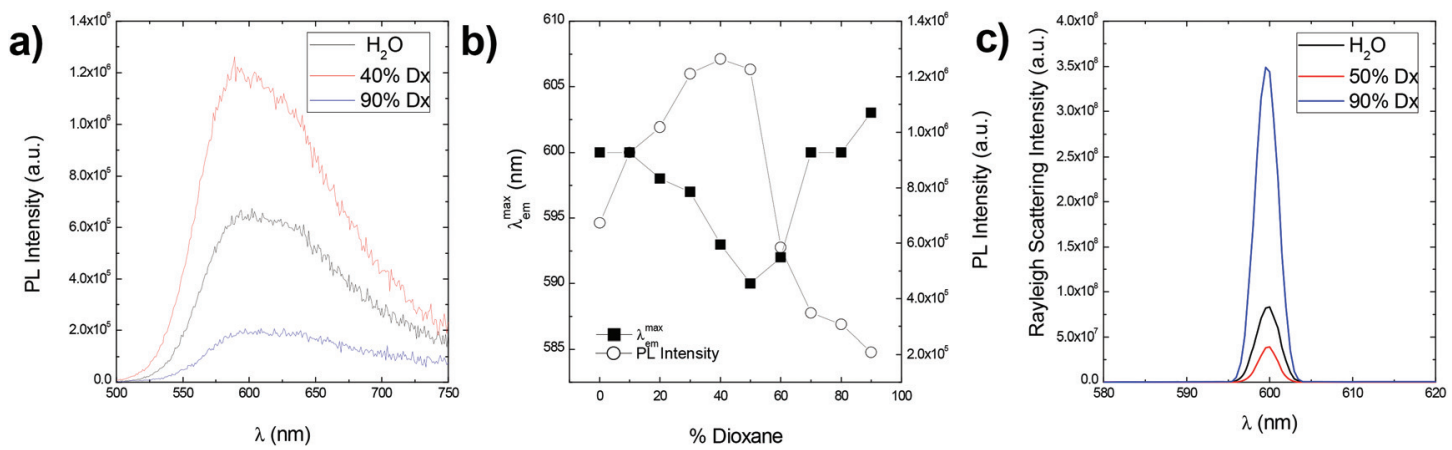

Fig. 1 (a) Photoluminescence (PL) spectra of P3SBDEAHT in dioxane : water (v/v) mixtures. (b) Dependence of the PL intensity and the emission maximum on the percentage of dioxane in dioxane : water (v/v) mixtures. (c) Rayleigh scattering peak (at $600 \mathrm{~nm}$ ) in water, $40 \%$ dioxane and $90 \%$ dioxane content in the mixtures.

content is between 30 and $50 \%(\mathrm{v} / \mathrm{v})$ the PL intensity is higher and the spectra are blue-shifted relative to both pure water and to the mixtures with high dioxane content. At higher dioxane contents the intensity drops, and a reverse red-shift is observed. The shifts and the changes in PL spectra can be explained by means of intermolecular interactions. ${ }^{34}$ It has been shown that the solvent effects on PL spectra are associated with the changes in the aggregation state of the solution, due to the different balance between hydrophobic and hydrophilic interactions in the mixtures. ${ }^{35-39}$ In water, interchain hydrophobic interactions ( $\pi-\pi$ interactions) are dominant and aggregates are formed to avoid contact between the hydrophobic chains of the polymer and water. The close proximity between the conjugated segments of different polymer chains allows interchain migration of photoexcitations and consequently emission from the lower energy-emitting sites occurs, resulting in lower PL and red-shifted emission. In 30-50\% dioxane mixtures, the optimum balance between hydrophobic and hydrophilic interactions is attained; interchain interactions are precluded and the PL intensity increases. At higher dioxane contents, new aggregated structures are formed. The decrease in the polarity of the solvent favours polymer backbone-solvent interactions. The ZCPE will orient itself and aggregate in order to avoid the less favourable solvent-zwitterionic chain interactions. Red-shifted PL is again observed. The aggregation/de-aggregation/aggregation process may be fol- 


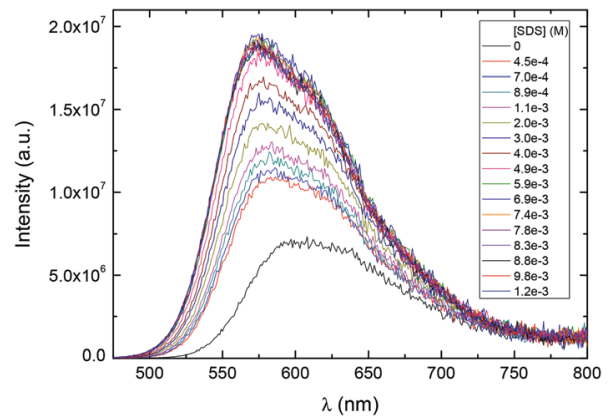

Fig. 2 PL spectra of P3SBDEAHT in aqueous solutions in the presence of SDS.

lowed by the intensity of the Rayleigh scattering peak (Fig. 1c). Its intensity decreases from water to $50 \%$ dioxane, and rises again in a $90 \%$ dioxane mixture. In fact, the scattering intensity is higher in $90 \%$ dioxane than in water, indicating the formation of larger aggregates in solution, due to the very low solubility in dioxane.

Similar effects were obtained with the addition of surfactants to an aqueous solution of P3SBDEAHT. The effect of nonionic $\left(\mathrm{S}^{0}\right)$, anionic $\left(\mathrm{S}^{-}\right)$, cationic $\left(\mathrm{S}^{+}\right)$and zwitterionic $\left(\mathrm{S}^{+/-}\right)$ surfactants was studied and a quite different behavior was found that depends on the nature of the charged surfactants. The addition of cationic surfactants, dodecyl trimethylammonium chloride (DTAC) and hexadecyltrimethyl- ammonium bromide (CTAB), caused similar effects on the optical properties of P3SBDEAHT (Fig. S1 $\uparrow$ and Table 1). Below the critical micelle concentration $(\mathrm{cmc})$ of the cationic surfactant, the intensity, the maximum and the shape of the PL spectra does not significantly change (see Fig. S1, in the ESI†). When the surfactant concentration approaches the cmc, we can identify an increase of PL intensity and a blue-shift (by $c a .6 \mathrm{~nm}$ ) of the spectra. The surfactant concentration at which these changes are observed corresponds to the critical aggregation concentration (cac). This concentration is, most probably, due to conformational changes of single P3SBDEAHT chains in P3SBDEAHT/anionic surfactant aggregates. Anionic sulfate surfactants (with chain lengths $n=8,12$ and 14, labeled as SOS, SDS and STS, respectively) induce different changes in the optical spectra of P3SBDEAHT when compared with cationic surfactants (Fig. 2 and 3). Upon addition of the anionic surfactant $\left(c_{\text {surf }} \ll \mathrm{cmc}\right)$, the PL intensity is first enhanced and the PL band is blue-shifted. The PL intensity continuously increases upon further addition of the surfactant. Starting at concentrations close to the cmc the PL intensity and emission maximum emission remain constant during further surfactant addition. In Fig. 3 one can identify two switching points for the dependence of PL intensity and $\lambda_{\max } v s$. surfactant concentration. The first break occurs at concentrations well below the $\mathrm{cmc}$ and may be due to some disaggregation of interchain aggregates (so-called critical de-aggregation concentration cac* in Table 1). The second turn corresponds to the cac, also observed in P3SBDEAHT/ $/ \mathrm{S}^{+}$surfactant systems.
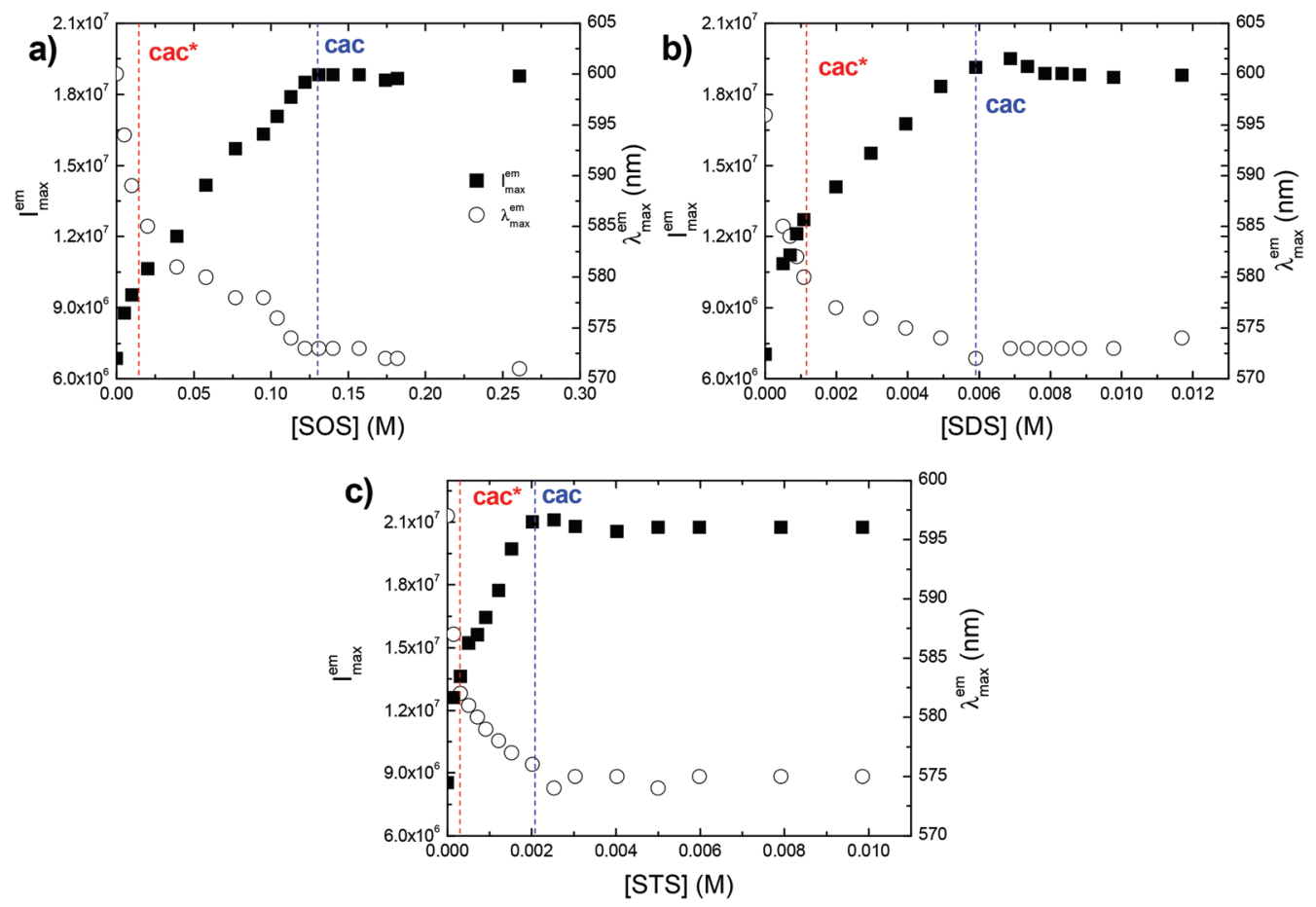

Fig. 3 Dependence of the PL intensity $(\square)$ and maximum emission wavelength $(O)$ of P3SBDEAHT on surfactant concentration for addition of anionic surfactants (a) SOS, (b) SDS and (c) STS. The dashed lines represent the transition concentrations: cac* (red line) and cac (blue line). 


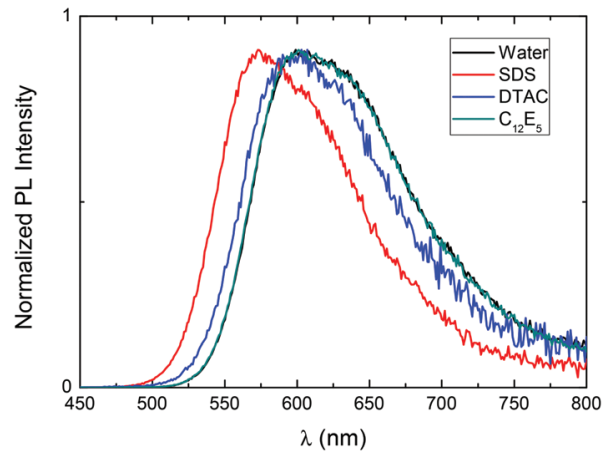

Fig. 4 PL emission spectra of P3SBDEAHT in aqueous solution containing surfactants with $C_{12}$ tails: P3SBDEAHT(SDS), P3SBDEAHT(DTAC) and P3SBDEAHT $\left(\mathrm{C}_{12} \mathrm{E}_{5}\right)$, at surfactant concentrations above the $\mathrm{cmc}$.

Comparing the optical responses of aqueous P3SBDEAHT solutions in the presence of single-tail surfactants with dodecyl chains (anionic SDS, cationic DTAC, and the non-ionic surfactant $\left.\mathrm{C}_{12} \mathrm{E}_{5}\right)$ the surfactochromic effect ${ }^{10}$ increases as follows: $\mathrm{C}_{12} \mathrm{E}_{5}<$ DTAC $<$ SDS (Fig. 4 and Table 1). The hydrophobic interactions of the surfactant tails with P3SBDEAHT should be similar for the three systems. Therefore, the observed differences reflect the influence of electrostatic interactions of the head groups of the surfactants and the ionic side chains of the ZCPE. In fact, for the P3SBDEAHT $\left(\mathrm{C}_{12} \mathrm{E}_{5}\right)$ system, no significant interaction was documented in the absorption and fluorescence spectra. However, this does not mean the absence of any interaction. Molecular dynamics simulations (MDS) provided evidence for the formation of P3SBDEAHT $\left(\mathrm{C}_{12} \mathrm{E}_{5}\right)$ mixed aggregates (see bellow). The different effect promoted by SDS and DTAC is related to the position of the positively and negatively charged groups in the zwitterionic side chain. SDS interacts specifically with diethylammonium groups and the hydrophobic tail of the surfactant moves towards the hydrophobic domains of the polyelectrolyte. In the case of cationic surfactant DTAC, the polymer-surfactant interactions are non-specific and occur at the outer part of the zwitterionic chain. The tail of the cationic surfactant is not deeply embedded into the P3SBDEAHT-surfactant aggregate, explaining the weaker response in the photophysical properties of the polymer. This will be discussed more in detail in the MDS section.

Addition of a zwitterionic surfactant, cocamidopropyl betaine (CAPB), to an aqueous solution of P3SBDEAHT leads to a gradual increase in the fluorescence intensity at concentrations slightly below the cmc, accompanied by a blue-shift of ca. $7 \mathrm{~nm}$ (see Fig. S2, in the ESI $\dagger$ ). The effect on the optical properties is similar to that found for the cationic surfactant. The zwitterionic side chains of the P3SBDEAHT should cause strong dipolar interactions with the zwitterionic head group of CAPB. Here, the formation of a zipper-like arrangement similar to that found in the hydrogelation of a zwitterionic poly(fluorene-phenylene) may occur. ${ }^{19}$ However, the distance between the positively and negatively charged centers in the CAPB surfactant is significantly smaller than that in P3SBDEAHT. Therefore, a zipper-like arrangement is not likely; the interaction should mainly occur at the periphery of the zwitterionic side chain, similar to the interaction mode postulated for cationic surfactants, in accordance with the similar response of the optical properties during surfactant addition.

\section{Electrical conductivity}

The interaction between P3SBDEAHT and ionic surfactants also shows a different behavior in electrical conductivity (EC) measurements. In the presence of P3SBDEAHT, the dependence of the electrical conductance $(\kappa)$ on surfactant concentration (Fig. 5) resembles the one obtained for the pure surfactant system, i.e., at pre-micelle concentrations. Generally, $\kappa$ increases with increasing surfactant concentration. At a certain concentration we observe a change in the slope: further increasing the surfactant concentration leads to a slower increase in $\kappa$. This turning point is associated with the cmc for the pure surfactant systems or with critical aggregation
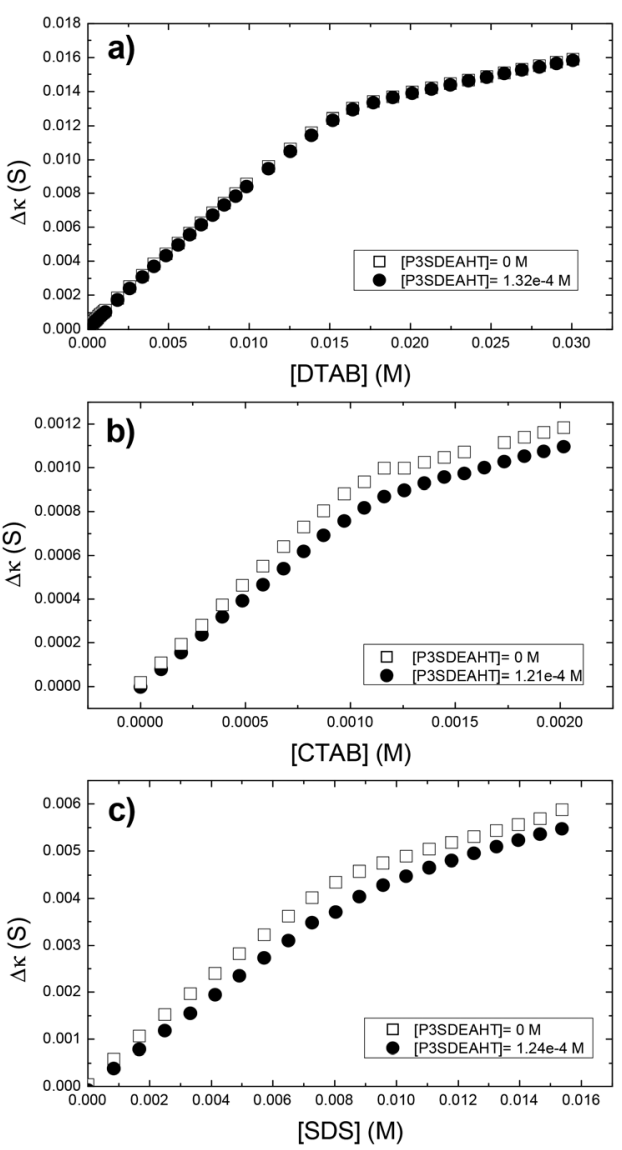

Fig. 5 Effect of the addition of DTAB (a), CTAB (b) and SDS (c) on the electrical conductance $(\Delta \kappa)$ of P3SBDEAHT, at $25^{\circ} \mathrm{C}$. The $\kappa$ of the pure surfactant systems are also shown. 
concentration (cac) in typical polymer-surfactant systems, respectively.

Interactions between cationic surfactants (DTAB and CTAB) and P3SBDEAHT show that the presence of the CPE does not change the cmc (Fig. 5a and b). However, a slight increase in the degree of dissociation of counterions is noted for CTAB. This change is enough to increase the Gibbs energy of micellization for $c a$. $1.5 \mathrm{~kJ} \mathrm{~mol}^{-1}$, suggesting that the polymer aggregation is dependent on the hydrocarbon chain length (reasons behind this increase in the $\Delta G^{0}$ algebraic value will be discussed below). On the basis of our MDS discussion, it is proposed that alkyltrimethyl ammonium surfactants do not significantly affect the aggregation of P3SBDEAHT and the interactions between surfactants and polymers mainly occur on the outer region of the polyelectrolyte aggregates. This suggests that the interactions are weaker and mainly driven by hydrophobic interactions, and, consequently, the EC response is very weak.

A significantly different behavior is observed with the SDS-P3SBDEAHT system. Comparing the effect of the polyelectrolyte on the free energy of micellization of SDS with that obtained for DTAB, one can conclude that for similar P3SBDEAHT concentrations, an anionic surfactant induces a more significant change in the formation of surfactant micelles $\left(-34.43 \mathrm{~kJ} \mathrm{~mol}^{-1}\right.$ vs. $-37.1 \mathrm{~kJ} \mathrm{~mol}^{-1}$ for pure SDS and $-36.00 \mathrm{~kJ} \mathrm{~mol}^{-1}$ vs. $-36.06 \mathrm{~kJ} \mathrm{~mol}^{-1}$ for pure DTAB). Such a change cannot be thermodynamically justified unless a given amount of SDS (labeled as [S]*) has been reacted with the polyelectrolyte in such a way that the effective cmc should be calculated by subtracting $[\mathrm{S}]^{*} .{ }^{42}$ To test the validity of this hypothesis, the dependence of cac on the polyelectrolyte concentration has been evaluated (see Table 2). A plot (not shown) of cac $=f[\mathrm{P} 3 \mathrm{SBDEAHT}]$ results in a linear dependence with a slope of $c a .15 .2\left(R^{2}=0.9987\right)$. A different point arises from this discussion: if there is an interaction between the ZCPE and the surfactant, it should be expected that a further turning point at pre-micelle concentrations of the surfactant should be detectable $^{43}$ (Fig. 5) as observed in the fluorescence measurements (Fig. 3). However, the two techniques monitor different physical quantities and, consequently, different structural phenomena. Whilst fluorescence is able to follow changes in the polymer backbone, the conductivity depends mainly on the charge density of ionic species in solution. From that it arises that most probably the interaction between SDS and the polyelectrolyte is characterized by weak electrostatic interactions and, consequently, cannot be monitored during electrical conductance measurements. This seems to agree with MDS studies where the presence of the anionic surfactant SDS breaks up the polyelectrolyte aggregates in contrast with the cationic DTAB, thus highlighting the importance of the kind of charge in the surfactant. This was corroborated by measuring the cac of SDS in aqueous solutions of P3SBDEAHT at different temperatures (Table 3). By increasing the temperature the electrostatic interactions between the negatively charged dodecylsulfate and the positively charged P3SBDEAHT increase and lead to an increasing ionic mobility and, conse- quently, to a gain in the free energy of micellization, computed according to the following equation:

$$
\Delta G_{\mathrm{mic}}=R T \ln \left(\frac{\mathrm{cac}}{\mathrm{cmc}}\right)
$$

EC measurements for the interaction between zwitterionic P3SBDEAHT and zwitterionic CAPB were also carried out but, as expected, no significant changes or turning points in the electrical conductance plots were observed for increasing surfactant concentration.

\section{SAXS}

Fig. 6 shows the plots of SAXS curves for zwitterionic P3SBDEAHT and P3SBDEAHT complexed with zwitterionic CAPB. Structural parameters estimated from these data are
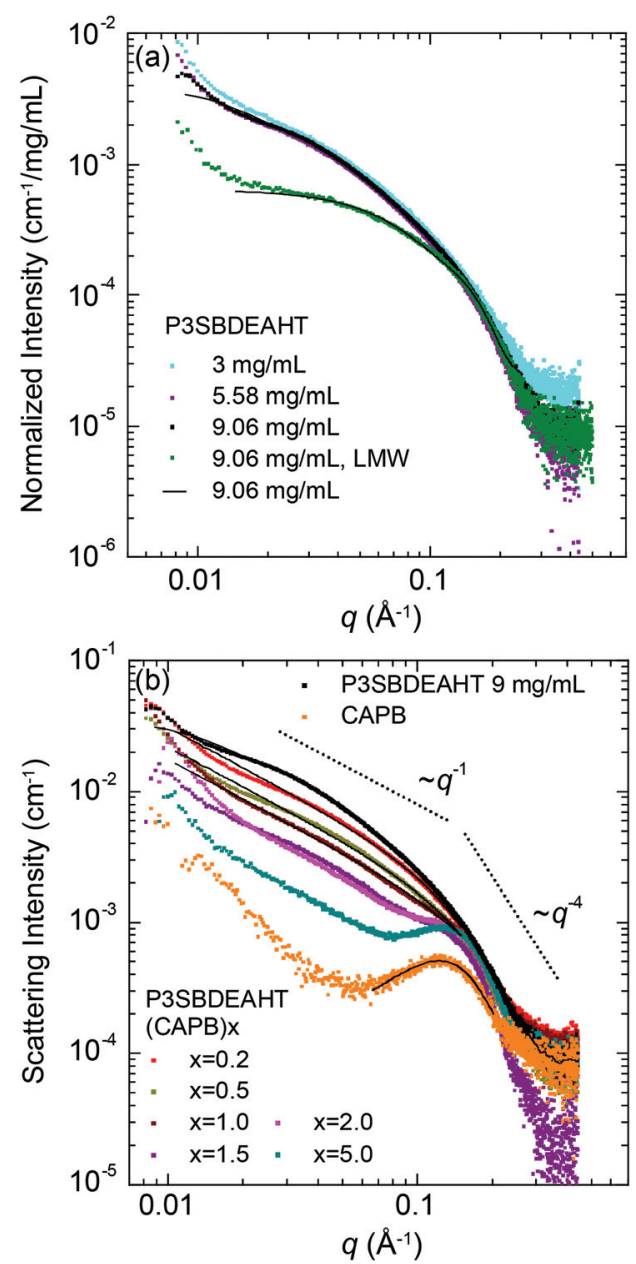

Fig. 6 (a) SAXS data of P3SBDEAHT in water at concentrations $3 \mathrm{mg}$ $\mathrm{mL}^{-1}$ (cyan squares), $5.58 \mathrm{mg} \mathrm{mL}^{-1}$ (purple) and $9.06 \mathrm{mg} \mathrm{mL}^{-1}$ (black) and $9.06 \mathrm{mg} \mathrm{mL}^{-1}$ low molecular weight P3SBDEAHT (olive). The curves are normalized to concentration. Solid line is the fit to the last data. (b) SAXS data of P3SBDEAHT(CAPB) $x$ with $x=0.2$ (red), $x=0.5$ (dark yellow), $x=1.0$ (wine), $x=1.5$ (violet), $x=2$ (magenta) and $x=5$ (cyan). Solid lines are fits to the data. Data of $9.06 \mathrm{mg} \mathrm{mL}^{-1}$ P3SBDEAHT (black) and CAPB (orange) and -1 and -4 decays are plotted for comparison. $T=$ $25^{\circ} \mathrm{C}$. 
compiled in Table 4. Fig. 6(a) shows the plots for P3SBDEAHT for a dilution series normalized to the sample concentration with a molecular weight of $23.4 \mathrm{~kg} \mathrm{~mol}^{-1}$. Also shown are data for P3SBDEAHT with a molecular weight of $11.5 \mathrm{~kg} \mathrm{~mol}^{-1}$. Regarding higher molecular weight polymers, smooth curves are leveling off at $\sim 0.01-0.03 \AA^{-1}$, which point to essentially 3-dimensional (3D) polymer aggregates with the radius of gyration of about $85 \AA$. Some emerging upturn at the lowest $q$ imply emerging larger aggregates. The data are essentially concentration independent and do not display interference maxima. These together imply that the particles are not interacting with each other. The scattering intensity of low molecular weight P3SBDEAHT is much lower and the particles are significantly smaller.

Fig. 6(b) shows the plots for P3SBDEAHT(CAPB $)_{x}$ for $x=$ 0.2-5. The curves for pure P3SBDEAHT and CAPB are shown for comparison. Like P3SBDEAHT, CAPB shows increasing scattering at low scattering angles pointing to the aggregation of surfactants. While P3SBDEAHT curves are featureless, CAPB is showing a distinctive interference maximum at $0.12 \AA^{-1}$ corresponding to the periodicity of $52 \AA$. It is not clear whether this peak stems from the internal structure of CAPB aggregates or from their electrostatic interactions.

Since both P3SBDEAHT and CAPB are zwitterionic, this combination allows nominal charge neutralization at $x=1$. The data of P3SBDEAHT(CAPB) $)_{x}$ differs from their constituents indicating the emergence of a new structural organization. For $q<0.15 \AA^{-1}$ the curve decays as $q^{-1}$ for all $x$. For $q>$ $0.15 \AA^{-1}$ the curves remain featureless scaling as $q^{-4}$. We interpret these observations as an existence of $\sim 20$ A thick cylindrical aggregates where all CAPB molecules are associated with the polymer. The emergence of cylindrical aggregates and no sign of interference maxima are analogous to the phase behavior of a polymer-surfactant pair with both constituents containing only one (but opposite) charge, when the molar fraction $x$ is screened over the nominal charge compensation point. ${ }^{8,9}$ Furthermore, these data are reminiscent to the rigid rod polyelectrolytes with strong clustering of counterions around the polymer chain. ${ }^{44}$ When the CAPB fraction is increased for $x>1$, the data show an interference maximum similar to that of pure CAPB. This is a sign of an emerging phase segregation of pure CAPB.

The P3SBDEAHT(SDS) $)_{x}$ system was also studied through SAXS (see Fig. S3 $\dagger$ ). For $x=0.2-1$ the scattering curve displays a broad peak at $q \approx 0.18 \AA^{-1}$. Free SDS micelles show a similar peak that stems from the polar cell of the surfactant heads ${ }^{45}$ and this points to the emerging phase separation even below $x=1$. This was confirmed through EC, where the break in the conductivity curves was assigned to the formation of pure SDS micelles co-existing with P3SBDEAHT-SDS unimer aggregates (Fig. 5c). Molecular dynamics simulations showed that these merge into large P3SBDEAHT-SDS aggregates (see below). The fact that these are not observed through SAXS may indicate that the size of the aggregates is out of our detection range. The structural organization of the P3SBDEAHT(SDS) $)_{x}$ system differs from that found with cationic P3TMAHT ${ }^{38,46}$ and $\mathrm{S}^{-}$surfactants, and may explain some of the optical differences found when we compare the ZCPE- and the cationic CPE-surfactant systems. The optical changes of cationic P3TMAHT(SDS) $)_{x}$ and P3TMAHT(SOS) systems are accompanied by phase transitions from charged P3TMAHT aggregates with interparticle order to rodlike and sheet-like particles with embedded polymer bundles or sheet-like polymer associations. The emergence of vibronic structures for charge ratios lower than $1 / 2$ and phase separation, i.e., dissolved and precipitated phases co-exist in equilibrium, at nominal charge compensation, are also observed. ${ }^{46}$ In the present study, the PL band is structureless in the range of $\mathrm{S}^{-}$concentration studied and phase separation was not observed. Independently of the charge of the surfactant, the optical properties of the system can be modulated by changing surfactant concentration without the risk of precipitation. Different surfactants will promote different chain conformations due to their different packing within the aggregate. This is an important property of these polymersurfactant systems from the solution processing point of view.

\section{Molecular dynamics simulations (MDS)}

Molecular dynamics simulations were performed to examine the aggregation of the tetrameric form of P3SBDEAHT in

Table 4 Essential structural parameters obtained from the SAXS data for P3SBDEAHT, low molecular weight (LMW) P3SBDEAHT, CAPB and P3SBDEAHT(CAPB $)_{x}$ in Water ${ }^{a}$

\begin{tabular}{|c|c|c|c|c|c|c|c|c|c|}
\hline P3SBDEAHT & 3.00 & & $0.009-0.43$ & - & $3 \mathrm{D}$ & 300 & $87.7 \pm 1.3$ & - & - \\
\hline P3SBDEAHT & 9.06 & & $0.009-0.43$ & - & 3D & 300 & $87.7 \pm 0.8$ & - & - \\
\hline P3SBDEAHT - LMW & 9.06 & - & $0.014-0.25$ & - & 3D & 60 & $21.1 \pm 0.1$ & - & \\
\hline P3SBDEAHT(CAPB) $x=0.2$ & 7.48 & 1.58 & $0.01-0.15$ & $1.14 \pm 0.02$ & Cylinder & 25 & - & $8.2 \pm 0.1$ & - \\
\hline
\end{tabular}

${ }^{a} \alpha, D_{\max }, R_{\mathrm{g}}$, and $R_{\mathrm{CS}, \mathrm{g}}$, are, respectively, the scattering power, considered maximum size of the particle (particle size or rod diameter) and the radius of gyration for the whole arbitrary-shaped particle or for the cross section of a cylindrical particle. Also shown is the position of an interference maximum for the CAPB sample. 

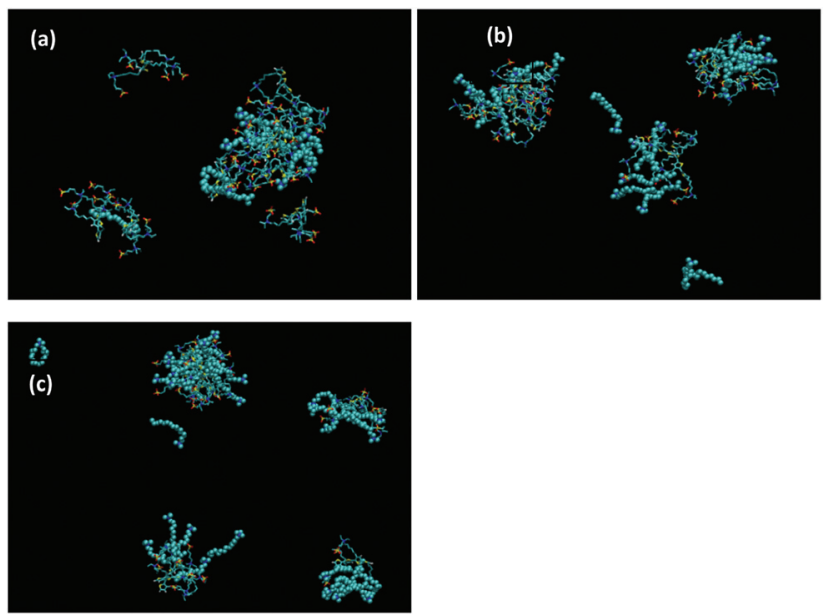

Fig. 7 Simulation cell of P3SBDEAHT and CTAB in (a) $1: 1$, (b) $1: 2$ and (c) $1: 3$ ratios. CTAB shown in van der Waal's representations.

surfactant $\left(\mathrm{S}^{+}, \mathrm{S}^{-}, \mathrm{S}^{0}\right.$ and $\left.\mathrm{S}^{+/-}\right)$environments. Simulation boxes were created according to the specifications shown in the Experimental section (ESI $\dagger$ ).

The simulation cells at $20 \mathrm{~ns}$ with different P3SBDEAHT : $\mathrm{S}^{+}$ surfactant (DTAC and CTAC) ratios show a clear interaction between the $\mathrm{S}^{+}$and the $\mathrm{ZCPE}$ (Fig. $\mathrm{S} 4 \dagger$ and 7, respectively). The interaction appears to occur predominantly on the outer region of the surfactant: P3SBDEAHT aggregates. The localiz-

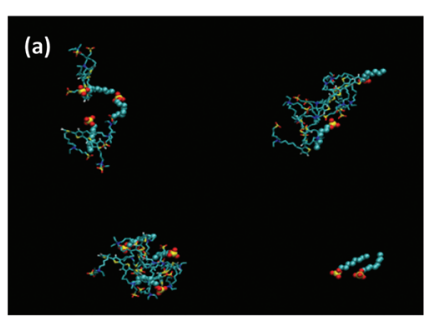

ation of the interaction with $\mathrm{S}^{+}$surfactants confirms the minimal effect on the polymer backbone, and hence on the effect on the conjugation length of the ZCPE.

Anionic surfactants with three different chain lengths (SOS, SDS and STS) had greater effect in the aggregation behavior of P3SBDEAHT tetramers, than $\mathrm{S}^{+}$. Fig. 8(a) shows the effects of the addition of SOS to P3SBDEAHT. Interaction clearly occurs at each of the three ratios although some solubility of the surfactant is apparent in each case. Comparable observations were found upon addition of SDS (Fig. 8(b)) although with a lesser amount of surfactant solubility is observed. The overall system does appear to be more disperse and it can be observed that aggregation of P3SBDEAHT is inhibited. At the highest surfactant: polyelectrolyte ratio this seems to result in the formation of a large surfactant-polyelectrolyte aggregate (see Fig. 8 right hand panels). A similar behaviour was also found for addition of STS, Fig. 8(c). At the lowest ratio there is a clear interaction between the P3SBDEAHT and STS. In this case the aggregates formed appear to be better defined than what was observed for SOS and SDS. As the ratio of the anionic surfactant to the electrolyte increases it becomes very clear that a rather specific interaction is occurring where the tail groups of the surfactant have become noticeably embedded in the surfactant/polyelectrolyte aggregate and the head groups remain exposed to the surrounding solvent.

In the presence of zwitterionic CAPB there is a clear interaction between the polyelectrolyte and the surfactant through the formation of mixed P3SBDEAHT(CAPB) aggregates
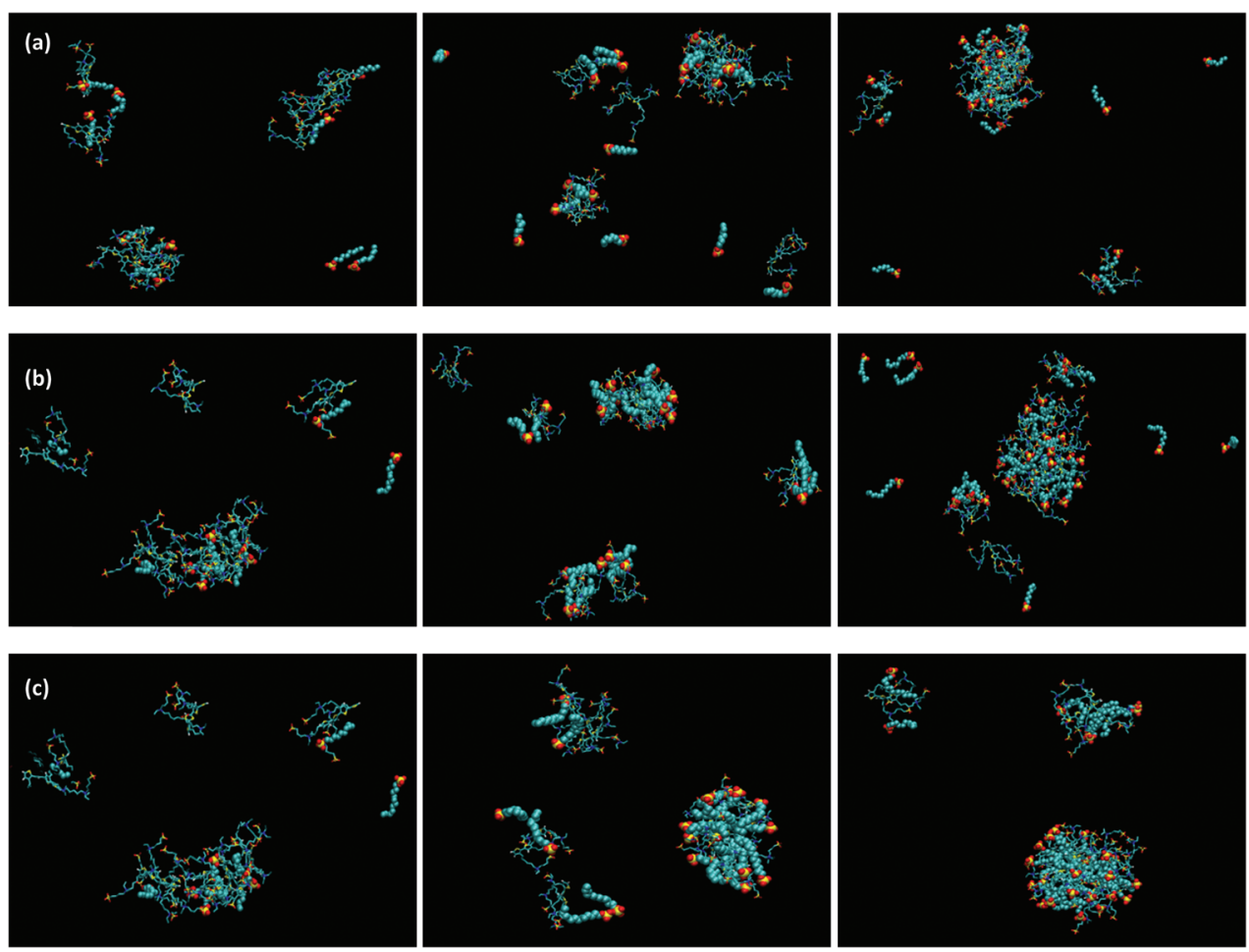

Fig. 8 Simulation cell of P3SBDEAHT and (a) SOS, (b) SDS and (c) STS in 1:1 (left hand panels), 1:2 (middle hand panels) and 1:3 (right hand panels) ratios. SOS, SDS and STS are shown in van der Waal's representations. 
(Fig. S5†). However, although it is extremely likely that those interactions are governed primarily to minimise unfavourable electrostatic interactions, it is possible that these reorientations do not significantly affect the structural arrangement of P3SBDEAHT and resultantly do manifest as minor changes in spectroscopy measurements.

Simulations of P3SBDEAHT and $\mathrm{C}_{12} \mathrm{E}_{5}$ show that interaction does occur between the polyelectrolyte and the surfactant (Fig. S6†) but it is possible that the hydrophobic tail group of the surfactant is too long to embed into the zwitterion aggregate sufficiently to cause any structural or electronic changes that could be detected by spectroscopy or photochemical techniques. This suggests that the interaction between the nonionic surfactant and the polyelectrolyte takes place predominantly on the outer part of the polyelectrolyte aggregates and has little effect on P3SBDEAHT.

\section{Conclusions}

The zwitterionic polymer P3SBDEAHT forms large aggregates in aqueous solutions. Experimental and theoretical studies allow for deriving a detailed picture on the interaction of the zwitterionic conjugated polyelectrolyte P3SBDEAHT with various surfactants and revealed that the nature of the surfactant (cationic $\mathrm{S}^{+}$, anionic $\mathrm{S}^{-}$, zwitterionic $\mathrm{S}^{+/-}$, and non-ionic $\mathrm{S}^{0}$ ) added to the solution of P3SBDEAHT has a significant effect on the photophysics of the zwitterion. This study emphasizes the importance of specific and non-specific interactions in the polymer-surfactant assemblies. The charge of the surfactant is, hereby, a key factor that governs the photophysics and the physicochemical behaviour of the zwitterionic P3SBDEAHT. Specific polymer-S ${ }^{-}$interactions occur between the triethylammonium groups of the zwitterionic side chains of the polyelectrolyte and the sulfate groups of the surfactant. To facilitate such ionic interactions the tail group of the surfactant has to be embedded into the polymer-surfactant aggregates, which induces conformational changes in the zwitterion backbone. Nevertheless, non-specific interactions seem to be very important in determining the position of the surfactant tail with respect to the aggregates and define the nanoscale organization of the system. These are especially important for the $\mathrm{S}^{+}$(DTAC and CTAB) and $\mathrm{S}^{+/-}$(CAPB) surfactants. Polymer$\mathrm{S}^{+}$and polymer- $\mathrm{S}^{+/-}$interactions arise from non-specific binding of the surfactant molecules to the peripheral, ionic side chains of P3SBDEAHT. In both cases, the majority of the $\mathrm{S}^{+}$and $\mathrm{S}^{+/-}$surfactant molecules are located outside of the polymer within the surrounding solvent with only a weak effect on the backbone arrangement. Small angle X-ray scattering experiments carried out on the P3SBDEAHT(CAPB) system showed a structural transition from 3D-aggregates of pure P3SBDEAHT to cylindrical mixed aggregates co-existing with an emerging phase segregation of CAPB micelles.

We believe our results are valuable in the field of conjugated polyelectrolytes: the combination of photophysical, molecular dynamics and small angle X-ray scattering analysis allows us to derive a detailed picture of the interaction of a zwitterion polythiophene with a range of surfactants. Recently, the occurrence of photoinduced electron-transfer cascades has been shown for poly(fluorene-alt-thiophene)/cationic fullerene assemblies leading to long-lived and stable polaron pairs. ${ }^{47}$ Finally, the detailed understanding of the surfactochromic behavior of conjugated (zwitter)ionic polyelectrolytes is of high importance for the design of new functional materials for use in solution processed optoelectronic devices and as biosensors.

\section{Acknowledgements}

The Coimbra Chemistry Centre is supported by the Fundação para a Ciência e a Tecnologia (FCT), Portuguese Agency for Scientific Research, through the project PEst-OE/QUI/UI0313/ 2014. TC and BS thank FCT, which has supported this work through Postdoctoral Grants (SFRH/BPD/47181/2008 and SFRH/BPD/82396/2011, respectively). DA acknowledges CNPq, Conselho Nacional de Desenvolvimento Científico e Tecnológico - Brasil, for financial support through the Grant "Bolsista do CNPq - Brasil". The research leading to the SAXS data has received funding from the European Community's Seventh Framework Programme (FP7/2007-2013) CALIPSO under grant agreement no. 312284 .

\section{Notes and references}

1 S. Das, D. P. Chatterjee, R. Ghosh and A. K. Nandi, RSC Adv., 2015, 5, 20160-20177.

2 H.-A. Ho, M. Boissinot, M. G. Bergeron, G. Corbeil, K. Dore, D. Boudreau and M. Leclerc, Angew. Chem., Int. Ed., 2002, 41, 1548-1551.

3 O. Inganäs, W. R. Salaneck, J.-E. Österholm and J. Laakso, Synth. Met., 1988, 22, 395-406.

4 J. Wang, Q. Zhang, K. J. Tan, Y. F. Long, J. Ling and C. Z. Huang, J. Phys. Chem. B, 2011, 115, 1693-1697.

5 X. Liu, Y. Tang, L. Wang, J. Zhang, S. Song, C. Fan and S. Wang, Adv. Mater., 2007, 19, 1471-1474.

6 M. Knaapila, T. Costa, V. M. Garamus, M. Kraft, M. Drechsler, U. Scherf and H. D. Burrows, Macromolecules, 2014, 47, 4017-4027.

7 M. Knaapila, T. Costa, V. M. Garamus, M. Kraft, M. Drechsler, U. Scherf and H. D. Burrows, J. Phys. Chem. $B, 2015,119$, 3231-3241.

8 M. Knaapila, R. C. Evans, V. M. Garamus, L. Almásy, N. K. Székely, A. Gutacker, U. Scherf and H. D. Burrows, Langmuir, 2010, 26, 15634-15643.

9 M. Knaapila, R. C. Evans, A. Gutacker, V. M. Garamus, N. K. Székely, U. Scherf and H. D. Burrows, Soft Matter, 2011, 7, 6863-6872.

10 J. J. Lavigne, D. L. Broughton, J. N. Wilson, B. Erdogan and U. H. F. Bunz, Macromolecules, 2003, 36, 7409-7412.

11 R. D. McCullough, P. C. Ewbank and R. S. Loewe, J. Am. Chem. Soc., 1997, 119, 633-634. 
12 J. Fang, B. H. Wallikewitz, F. Gao, G. Tu, C. Muller, G. Pace, R. H. Friend and W. T. S. Huck, J. Am. Chem. Soc., 2011, 133, 683-685.

13 C. Duan, L. Wang, K. Zhang, X. Guan and F. Huang, Adv. Mater., 2011, 23, 1665-1669.

14 A. Kumar, G. Pace, A. A. Bakulin, J. Fang, P. K. H. Ho, W. T. S. Huck, R. H. Friend and N. C. Greenham, Energy Environ. Sci., 2013, 6, 1589-1596.

15 U. Scherf, Angew. Chem., Int. Ed., 2011, 50, 5016-5017.

16 F. Liu, Z. A. Page, V. V. Duzhko, T. P. Russell and T. Emrick, Adv. Mater., 2013, 25, 6868-6873.

17 K. Peter, R. Nilsson, J. Rydberg, L. Baltzer and O. Inganas, Proc. Natl. Acad. Sci. U. S. A., 2003, 100, 10170-10174.

18 K. F. Karlsson, P. Asberg, K. Peter, R. Nilsson and O. Inganäs, Chem. Mater., 2005, 17, 4204-4211.

19 E. Elmalem, F. Biedermann, M. R. J. Scherer, A. Koutsioubas, C. Toprakcioglu, G. Biffid and W. T. S. Huck, Chem. Commun., 2014, 50, 8930-8933.

20 U. Scherf, S. Adamczyk, A. Gutacker and N. Koenen, Macromol. Rapid Commun., 2009, 30, 1059-1065.

21 M. Kraft, S. Adamczyk, A. Polywka, K. Zilberberg, C. Weijtens, J. Meyer, P. Görrn, T. Riedl and U. Scherf, ACS Appl. Mater. Interfaces, 2014, 6, 11758-11765.

22 L. Zhai and R. D. McCullough, Adv. Mater., 2002, 14, 901905.

23 A. C. F. Ribeiro, A. J. M. Valente, V. M. M. Lobo, E. F. G. Azevedo, A. M. Amado, A. M. Amorim da Costa, M. L. Ramos and H. D. Burrows, J. Mol. Struct., 2004, 703, 93-101.

24 J. Barthel, F. Feuerlein, R. Neuder and R. Wachter, J. Solution Chem., 1980, 9, 209-219.

25 D. I. Svergun, J. Appl. Crystallogr., 1992, 25, 495-503.

26 H. J. C. Berendsen, D. V. d. Spoel and R. v. Druren, Comput. Phys. Commun., 1995, 91, 43-56.

27 E. Lindahl, B. Hess and D. V. d. Spoel, J. Mol. Model., 2001, 7, 306-317.

28 C. Oostenbrink, A. Villa, A. E. Mark and W. F. V. Gunsteren, J. Comput. Chem., 2004, 25, 1656-1676.

29 M. D. Hanwell, D. E. Curtis, D. C. Lonie, T. Vandermeersch, E. Zurek and G. R. Hutchison, J. Cheminf., 2012, 4, 1-17.

30 A. K. Malde, L. Zuo, M. Breeze, M. Stroet, D. Poger, P. C. Nair, C. Oosterbrink and A. E. Mark, J. Chem. Theory Comput., 2011, 7, 4026-4037.

31 H. J. C. Berendsen, J. P. M. Postra, W. F. V. Gunsteren and J. Hermans, in Intermolecular Forces, ed. B. Pullman, Reidel, Dordrecht, 1981, pp. 331-342.
32 B. Hess, H. Bekker, H. J. C. Berendsen and J. G. E. M. Fraaije, J. Comput. Chem., 1997, 18, 463477.

33 N. C. F. M. M. A. B. VMD owned by the Theoretical and Computational Biophysics Group, at the Beckman Institute, University of Illinois at Urbana-Champaign.

34 H. D. Burrows, S. M. Fonseca, C. L. Silva, A. A. C. C. Pais, M. J. Tapia, S. Pradhan and U. Scherf, Phys. Chem. Chem. Phys., 2008, 10, 4420-4428.

35 S. Wang and G. Bazan, Chem. Commun., 2004, 25082509.

36 A. Gutacker, S. Adamczyk, A. Helfer, L. E. Garner, R. Evans, S. M. Fonseca, M. Knaapila, G. C. Bazan, H. D. Burrows and U. Scherf, J. Mater. Chem., 2010, 20, 1423-1430.

37 A. Gutacker, N. Koenen, U. Scherf, S. Adamczyk, J. Pina, S. M. Fonseca, A. J. M. Valente, R. C. Evans, J. S. de Melo, H. D. Burrows and M. Knaapila, Polymer, 2010, 51, 18981903.

38 M. Knaapila, R. C. Evans, A. Gutacker, V. M. Garamus, M. Torkkeli, S. Adamczyk, M. Forster, U. Scherf and H. D. Burrows, Langmuir, 2010, 26, 5056-5066.

39 S.-C. Liao, C.-S. Lai, D.-D. Yeh, M. H. Rahman, C.-S. Hsu, H.-L. Chen and S.-A. Chen, React. Funct. Polym., 2009, 69, 498-506.

40 K. Holmberg, B. Jonsson, B. Kronberg and B. Lindman, Surfactants and Polymers in Aqueous Solutions, John Willey \& Sons, West Sussex, 2nd edn, 2003.

41 R. S. Becker, J. S. Seixas de Melo, A. L. Macanita and F. Elisei, J. Phys. Chem., 1996, 100, 18683-18695.

42 A. J. M. Valente, H. D. Burrows, R. F. Pereira, A. C. F. Ribeiro, J. L. G. C. Pereira and V. M. M. Lobo, Langmuir, 2006, 22, 5625-5629.

43 M. J. Tapia, M. Monteserín, A. Costoyas, H. D. Burrows, A. T. Marques, A. A. C. C. Pais, A. J. M. Valente, R. Mallavia, U. Scherf, A. Pinazo, L. Pérez and M. C. Morán, J. Mol. Liq., 2010, 156, 18-27.

44 B. Guilleaume, J. Blaul, M. Wittemann, M. Rehahn and M. Ballauff, J. Phys.: Condens. Matter, 2000, 12, A245A251.

45 T. Zemb and P. Charpin, J. Physique, 1985, 46, 249-256.

46 R. C. Evans, M. Knaapila, N. Willis-Fox, M. Kraft, A. Terry, H. D. Burrows and U. Scherf, Langmuir, 2012, 28, 1234812356.

47 R. C. Huber, A. S. Ferreira, R. Thompson, D. Kilbride, N. S. Knutson, S. Devi, D. B. Toso, J. R. Challa, Z. H. Zhou, Y. Rubin, B. J. Schwartz and S. H. Tolbert, Science, 2015, 348, 1340-1343. 